\title{
Asymptomatic Ischemic Heart Disease in a 45-year-old Male Athlete: A Case Report
}

\section{Massimo Bolognesi $i^{1 *}$ and Diletta Bolognesi ${ }^{2}$}

${ }^{1}$ Sports Cardiology Medicine Centre, Asl 112 District of Cesena, Via Ungaretti 49447521 Cesena, Italy

${ }^{2}$ Asl 112 District of Cesena, Via Lambruschini 30747521 Cesena, Itlay

\begin{abstract}
Background: The primary purpose of pre-participation screening is identifying the cohort of athletes affected by unsuspected cardiovascular diseases, such as coronary artery disease (CAD) as well as preventing sudden cardiac death (SCD) during sports competitions or training, through appropriate intervention.

Case Report: This case report describes the recent history of a middle-aged marathon runner suffering from asymptomatic ischemic heart disease at high risk for SCD and illustrates the clinical pathway. An exercise ECG stress testing was positive for subendocardial ischemia. Computerized tomography (CT) coronary angiogram subsequently showed critical narrowing of the left anterior descending artery. Invasive coronary angiography was performed following angioplasty procedure. One month later an exercise ECG stress testing was normal, indicative of complete myocardial revascularization.

Conclusions: Silent myocardial ischemia (SMI) is not such a rare event in athletes, especially in endurance sports athletes. In fact, even though they are asymptomatic this does not exclude the presence of a severe coronary artery disease. Exercise stress testing is safe and affordable non-invasive screening for detecting asymptomatic coronary artery disease in athletes. Myocardial revascularization procedure is to re-establish the athlete's state of fitness for competitive sports.
\end{abstract}

Keywords: Asymptomatic marathon runner; Sport pre-participation screening; Coronary artery disease; Exercise ECG stress testing; Myocardial revascularization

\section{Introduction}

Systematic sports pre-participation screening of athletes has the potential to identify those at risk and reduce cardiovascular event mortality [1-3], although this does not always take place. The vast majority of 'at risk-athletes' does not experience pre- monitory symptoms thus, pre-participation screening represents the only strategy capable in identifying any underlying cardiovascular disorder. The importance of early identification in silent cardiovascular diseases at a sub-clinical stage relies on the concrete possibility of preventing sudden cardiac death (SCD) through not only life- style modification, which includes restriction of competitive sports activity, but also prophylactic treatment with drugs and implantable defibrillator. The primary cause of SCD is often an underlying disease that is clinically silent and unlikely to be suspected or diagnosed through spontaneous symptoms. Normally, general population high-physical activity levels are associated with reduced risk of asymptomatic coronary artery disease. There are however some reports of exercise-related sudden death and myocardial infarction in aerobically trained athletes [4,5]. Silent myocardial ischemia is not such a rare event in athletes, indeed quite the opposite. Even though athletes are asymptomatic, the possibility that they are suffering from severe coronary artery disease [6] cannot be excluded. There is a heated argument among sport cardiologists in Europe and the USA regarding the efficacy, impact of false-positive results, and cost- effectiveness in routine preparticipation cardiovascular screening of athletes $[7,8]$. Despite this discussion, it is clear that the objective of systematic pre-participation screening is to identify those athletes considered at-risk withdrawing them from training and competition with the goal of reducing the eventuallity of sudden cardiac death, and when appropriate [9] adopting preventive interventions. Pre-participation screening in this population is finalized to the identification of cardiovascular diseases such as silent myocardial ischemia (SMI) and coronary artery disease that have low incidence in the general population [10]. The precise methodology by which pre-participation screening is to be (or can be) performed has been the source of considerable debate and controversy [11]. Therefore, European protocol, as proposed by two European institutions, represents a welcome and important effort which has the potential of identifying previously unrecognized but lethal cardiovascular events during sport activities. The initiative has been created in order to increase awareness and interest in this clinical problem within the European community. This case report highlights the importance of exercise ECG stress testing in asymptomatic middleaged healthy athletes with exercise induced-myocardial ischemia and decide the best time for a qualified therapeutic choice when any ventricular re-polarization abnormalities, such as significative ST segment depression, are detected during sports pre-participation screening.

\section{Case Report}

This case report describes the history of a 45 -year-old male top marathon runner referred to the sports Cardiology Medicine Centre, in

*Corresponding author: Massimo Bolognesi, Sports Cardiology Medicine Centre, Asl 112 District of Cesena, Via Ungaretti 49447521 Cesena, Italy, Tel: +390547303240; E-mail: massbolo1@tin.it

Received November 11, 2013; Accepted December 23, 2013; Published January 04, 2014

Citation: Bolognesi M, Bolognesi D (2014) Asymptomatic Ischemic Heart Disease in a 45-year-old Male Athlete: A Case Report. J Gen Pract 2: 139. doi: 10.4172/2329-9126.1000139

Copyright: ( 2014 Bolognesi M, et al. This is an open-access article distributed under the terms of the Creative Commons Attribution License, which permits unrestricted use, distribution, and reproduction in any medium, provided the original author and source are credited. 
Citation: Bolognesi M, Bolognesi D (2014) Asymptomatic Ischemic Heart Disease in a 45-year-old Male Athlete: A Case Report. J Gen Pract 2: 139. doi: $10.4172 / 2329-9126.1000139$

Page 2 of 4

Cesena, Italy, for cardiologic evaluation after the detection of ventricular re-polarization abnormalities, such as ST depression in the ECG stress testing, found elsewhere during sports pre-participation screening and considered as a false positive test. The athlete was apparently healthy. Physical examination resulted average and as for chest pain, syncope and palpitations the athlete was asymptomatic. Only family history was positive for coronary artery disease (his father had suffered from myocardial infarction from a young age). Other cardiovascular risk factors, such as dyslipidemia, smoking or diabetes, were absent and the probability pre-test was low. The athlete was under training and in competitions for over five years. Resting ECG was normal (Figure 1 Panel A), while the ECG treadmill stress testing with Astrand protocol was positive for signs of impaired coronary flow reserve with high work load corresponding to $15 \mathrm{METs}$ and nearly $55 \mathrm{ml} / \mathrm{Kg} / \mathrm{min}$ of maximum oxygen uptake. In particular, the ECG showed horizontal ST - segment depression in the anterior precordial leads V4 toV6 (Figure 1 Panel B). Computerized tomography (CT) coronary angiogram subsequently showed calcification with critical narrowing of the proximal tract of the left anterior descending artery (LAD) (Figure 2). Computerized tomography (CT) coronary angiogram showed calcification with critical narrowing of the proximal tract of the left anterior descending artery confirmed both anatomically and functionally (using fractional flow reserve -FFR $<0.75$-) critical stenosis (Figure 3) of the left anterior descending artery that was treated with angioplasty and drug eluting stent. The invasive procedure resulted in an optimal vessel dilation and complete revascularization as can be seen in Figure 4. Thirty days later, a maximal exercise ECG treadmill stress testing resulted normal.

\section{Discussion}

The main goal of pre-participation screening is to identify the cohort of athletes affected by cardiovascular diseases at risk of sudden death during sports. Systematic pre-participation cardiovascular screening, primarily to exclude atherosclerotic coronary artery disease in Master athletes, is not customary practice. Such persons are usually

\section{Electrocardiographyc Findings}
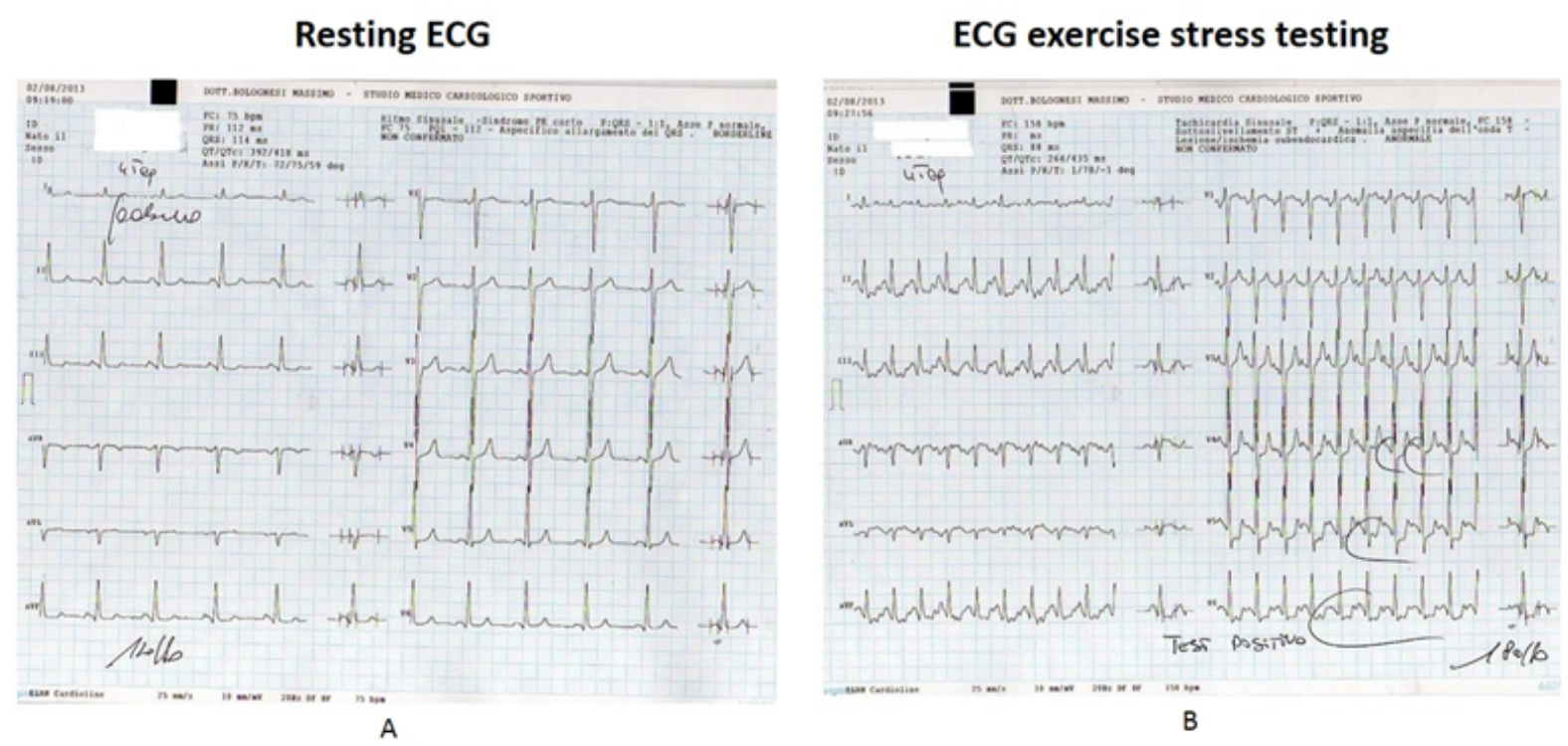

Figure1: Shows resting normal ECG in Panel A and exercise ECG treadmill stress testing with ST depression in precordial leads in Panel B.

\section{Proximal LAD artery stenosis $>70 \%$}
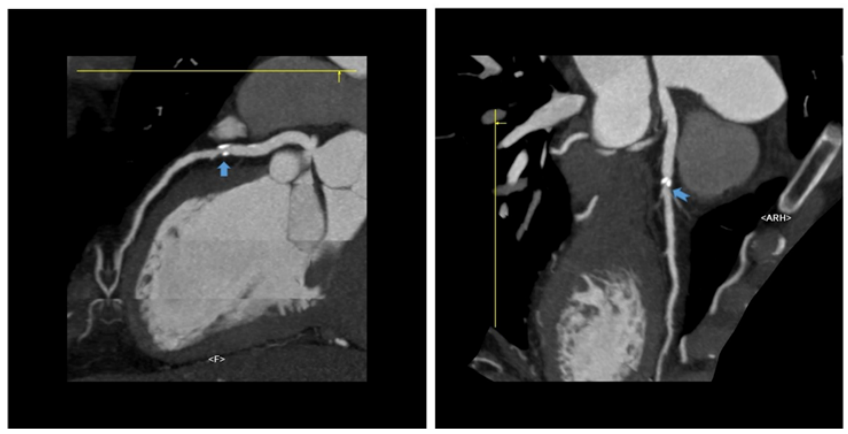

Figure 2: Shows computerized tomography (CT) coronary angiogram with critical narrowing (see arrows) of the proximal tract of left anterior descending artery. 
coronary angiogram before angioplasty
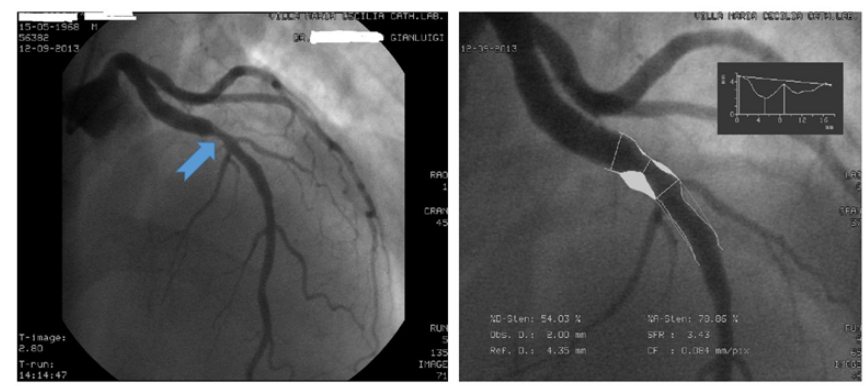

Figure 3: Shows coronary angiography findings (see arrows) on the LAD artery before angioplasty.

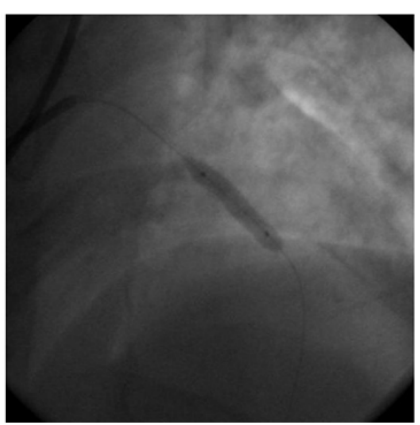

Figure 4: Shows coronary angiography after angioplasty with stent. participants in individual athletic activities such as road and marathon racing, or in a variety of other organized Master's sports [12]. The most common cause of cardiovascular events in athletes aged over35 years is ischemic heart disease. Silent myocardial ischemia is increasingly recognized as a common phenomenon among a variety of people with coronary artery disease, including competitive athletes. Little is known about incidence, threshold, and predictors of prognostically relevant silent ischemia. Cohn [13] divided SMI into three categories:

- Type I: This is the least common form and occurs in completely asymptomatic patients with CAD (which may be severe) in the absence of anginal symptoms.

- Type II: This type occurs in patients with documented previous myocardial infarction.

- Type III: This is the most common occurring in patients with the usual forms of chronic stable angina, unstable angina and vasospastic angina.

This case report highlights how silent myocardial ischemia is a condition that can also occur in middle-aged asymptomatic athletes when they present signs of subendocardial ischemia during exercise ECG testing, such as ST-segment depression. This finding should never be underestimated because silent electrocardiographic ST change predicts future coronary events in patients with CAD, i.e. SCD. This report emphasizing the value of exercise testing to identify asymptomatic high-risk athletes who could benefit from preventive measures [14]. Therefore, this case illustrates the ideal process and criteria to follow in sports medicine when signs of exercise-inducible ischemia in asymptomatic athletes during exercise stress testing are present. Data from the test, other than ECG changes, should be taken into consideration when interpreting the exercise stress test since it has prognostic value [15]. This case report is not unique or anecdotal, but rather represents the most recent experience of the authors $[16,17]$ in the last 20 years concerning early detection of silent ischemic heart disease in professional athletes during sports pre-participation screening. The rate of disqualification at pre-participation screening due to cardiovascular diseases is higher in Masters than younger athletes, with a greater incidence of ischemic heart disease [18].

\section{Conclusions}

Athletes with ischemic heart disease had known cardiovascular risk factors. Absence of symptoms, such as chest pain, is not a discriminating factor in the presence of severe ischemic heart disease, especially when the exercise ECG stress testing is positive for sign of impaired coronary flow reserve. Our report confirms the absence of symptoms and signs in Master athletes with atherosclerotic coronary disease and the importance of carefully investigating athletes with known cardiovascular risk factors during pre-participation screening, and the need to refer athletes for further clinical and instrumental investigation even though the exercise test is ambiguous.

\section{References}

1. Corrado D, Basso C, Schiavon M, Thiene G (1998) Screening for hypertrophic cardiomyopathy in young athletes. N Engl J Med 339: 364-369. 
Citation: Bolognesi M, Bolognesi D (2014) Asymptomatic Ischemic Heart Disease in a 45-year-old Male Athlete: A Case Report. J Gen Pract 2: 139. doi: 10.4172/2329-9126.1000139

Page 4 of 4

2. Pelliccia A, Maron BJ (1995) Preparticipation cardiovascular evaluation of the competitive athlete: perspectives from the 30-year Italian experience. Am J Cardiol 75: 827-829.

3. Corrado D, Basso C, Pavei A, Michieli P, Schiavon M, et al. (2006) Trends in sudden cardiovascular death in young competitive athletes after implementation of a preparticipation screening program. JAMA 296: 1593-1601.

4. Katzel LI, Fleg JL, Busby-Whitehead MJ, Sorkin JD, Becker LC, et al. (1998) Exercise-induced silent myocardial ischemia in master athletes. Am J Cardio 81: 261-265.

5. Maron BJ, Poliac LC, Roberts WO (1996) Risk for sudden cardiac death associated with marathon running. J Am Coll Cardiol 28: 428-431.

6. Deedwania PC, Carbajal EV (1991) Silent myocardial ischemia. A clinical perspective. Arch Intern Med 151: 2373-2382.

7. Corrado D, Pelliccia A, Bjornstad HH, Vanhees L, Biffi A, et al. (2005) Cardiovascular pre-participation screening of young competitive athletes for prevention of sudden death: proposal for a common European protocol. Consensus Statement of the Study Group of Sport Cardiology of the Working Group of Cardiac Rehabilitation and Exercise Physiology and the Working Group of Myocardial and Pericardial Diseases of the European Society of Cardiology. Eur Heart J 26: 516-524.

8. Maron BJ, Thompson PD, Ackerman MJ, Balady G, Berger S, et al. (2007) Recommendations and considerations related to preparticipation screening for cardiovascular abnormalities in competitive athletes: 2007 update: a scientific statement from the American Heart Association Council on Nutrition, Physical Activity, and Metabolism: endorsed by the American College of Cardiology Foundation. Circulation 115: 1643-1655

9. Maron BJ, Shen WK, Link MS, Epstein AE, Almquist AK, et al. (2000) Efficacy of implantable cardioverter-defibrillators for the prevention of sudden death in patients with hypertrophic cardiomyopathy. N Engl J Med 342: 365-373.
10. Maron BJ, Thompson PD, Puffer JC, McGrew CA, Strong WB, et al. (1996) Cardiovascular preparticipation screening of competitive athletes. A statement for health professionals from the sudden death committee (clinical cardiology) and congenital cardiac defects committee (cardiovascular disease in the young), American Heart Association. Circulation 94: 850-856.

11. International Olympic Committee (2004) Sudden cardiovascular death in sport. Lausanne Recommendations adopted 9-10 December 2004, Lausanne, Switzerland.

12. Maron BJ, Araújo CG, Thompson PD, Fletcher GF, De Luna AB, et al (2001) Recommendations for preparticipation screening and the assessment of cardiovascular disease in masters athletes: an advisory for healthcare professionals from the working groups of the World Heart Federation, the International Federation of Sports Medicine, and the American Heart Association Committee on Exercise, Cardiac Rehabilitation, and Prevention. Circulation 103: 327-334.

13. Cohn PF (1985) Silent myocardial ischemia: classification, prevalence, and prognosis. Am J Med 79: 2-6.

14. Laukkanen JA, Mäkikallio TH, Rauramaa R, Kurl S (2009) Asymptomatic ST segment depression during exercise testing and the risk of sudden cardiac death in middle-aged men: a population-based follow-up study. Eur Heart $J$ 30: $558-565$

15. Kharabsheh SM, Al-Sugair A, Al-Buraiki J, Al-Farhan J (2006) Overview of exercise stress testing. Ann Saudi Med 26: 1-6.

16. Bolognesi M, Bolognesi D (2014) Silent Myocardial Ischemia in Master Marathon Runners. Br J Med Med Res 4: 515-521.

17. Bolognesi M (2009) La Valutazione Medico Sportiva Agonistica nell'Atleta Master. Giornaleitaliano di cardiologia 6: 61-70.

18. Maron BJ, Zipes DP (2005) 36th Bethesda Conference: recommendations for determining eligibility for competition in athletes with cardiovascular abnormalities. J Am Coll Cardiol 45: 1373-1375. 